\title{
Renormalization Group Equations in Broken Supersymmetric Theories Using Superspace Methods
}

\author{
Asim Gangopadhyaya \\ Loyola University Chicago, agangop@luc.edu \\ Darwin Chang \\ University of Maryland - College Park
}

Follow this and additional works at: https://ecommons.luc.edu/physics_facpubs

Part of the Quantum Physics Commons

\section{Recommended Citation}

Chang, D. and Gangopadhyaya, A. (1986). Renormalization group equations in broken supersymmetric theories using superspace methods. Physical Review D, 33(6), 1771-1776.

This Article is brought to you for free and open access by the Faculty Publications and Other Works by Department at Loyola eCommons. It has been accepted for inclusion in Physics: Faculty Publications and Other Works by an authorized administrator of Loyola eCommons. For more information, please contact ecommons@luc.edu.

\section{(c) (i) $९$}

This work is licensed under a Creative Commons Attribution-Noncommercial-No Derivative Works 3.0 License.

(c) The American Physical Society, 1986. 


\title{
Renormalization-group equations in broken supersymmetric theories using superspace methods
}

\author{
Darwin Chang \\ Department of Physics, University of Maryland, College Park, Maryland 20742 \\ Asim Gangopadhyaya \\ Department of Physics, Central Michigan University, Mt. Pleasant, Michigan 48859 \\ and Department of Physics, City College of New York, New York, New York 10031
}

(Received 15 April 1985)

\begin{abstract}
We apply the superfield method with the spurion technique to derive the renormalization-group equations in broken supersymmetric theories. We point out some possible ambiguities in this procedure and show that it is in general necessary to express the supersymmetry-breaking terms in explicit $D$-type form. We also found that it is possible to construct broken supersymmetric theories where some of the symmetry-breaking parameters do not receive any infinite renormalization.
\end{abstract}

\section{INTRODUCTION}

Supersymmetry $^{1}$ (SUSY) has recently drawn a lot of attention from model builders in particle physics. Besides its aesthetic beauty of unifying bosons and fermions in one symmetry multiplet, it also provides a possible mechanism for solving ${ }^{2}$ the gauge hierarchy problem ${ }^{3}$ in grand unified theories and its gauged version is our first hope of having a normalizable unified theory of gravity and strong and electroweak interactions.

In global supersymmetric theories, one of the most amazing features is the cancellation between bosonic and fermionic loop contributions first observed by Wess and Zumino. As a result of this all quadratic divergences vanish from the theory and only logarithmic infinities survive. ${ }^{4}$ A SUSY multiplet that includes bosonic and fermionic components can most easily be represented by a function in superspace called a superfield. ${ }^{5}$ Superspace consists of four ordinary spacetime and four Grassmannian coordinates. Superfields, besides providing an elegant formalism, are also a very useful tool in practical calculations. The superfield Feynman rules have been formulated and improved upon in the literature (for a review, see Ref. 6). However, relatively less attention has been paid to the application of superfield formalism to calculations in realistic models. The reason is quite obvious. There is no evidence of SUSY in our elementary-particle-physics phenomenology. If SUSY is relevant to nature it must be broken one way or another at the energy scale we have been observing so far. Apparently the superfield formalism, as it is, must be modified to take into account the effect of the SUSY breaking. A very simple artifact, called the spurion ${ }^{7}$ technique, exists in the literature for this purpose. Since in the superfield language SUSY transformation is equivalent to translation in superspace, giving some superfield $\Phi(x, \theta, \bar{\theta})$ a fixed nonzero value results in SUSY breaking. The procedure is to introduce some external (spurion) superfields and let them couple to the quantum fields in SUSY fashion. The SUSY breaking is then achieved by giving the spurion a suitable chosen value. A
SUSY breaking is called soft breaking (SSB) if it does not generate any quadratic divergence. The soft terms preserve the nice ultraviolet properties of SUSY theory while making it realistic. Girardello and Grisaru have used the spurion technique to analyze all possible softbreaking terms in $N=1$ SUSY theories. ${ }^{7}$ The spurion technique is very useful in learning about the divergence structure of the realistic models. When one applies it to calculate a physical quantity, say, the $\beta$ function of a coupling constant, a question arises. Given a soft-SUSYbreaking term in the Lagrangian, there is more than one way it can be written in the superfield form with spurions. All the superfield forms that correspond to the same term in components, are equivalent. However, some of them are more convenient and economical than others. It is the purpose of this paper to shed light on this question and demonstrate it through toy models and realistic applications. In Sec. II we illustrate the ambiguity in choosing soft-breaking terms in a toy example and demonstrate the way to fix it. In Sec. III we show that all the soft-SUSYbreaking terms in a theory with supersymmetric interaction can be and should be written as $D$-type terms. This is the main result of the paper. The $F$-type terms that give rise to the same component forms can be discarded because they will automatically generate the corresponding $D$ terms with divergence coefficients at higher orders. Therefore, the inclusion of $F$ terms is redundant once the right $D$ terms are chosen. In the Appendix we demonstrate an application of this approach in a realistic model $-N=1$ supergravity model with a SUSY-breaking hidden sector. We derive the renormalization-group equations (RGE's) in this model using our approach and spell out the specific supergraphs that contribute to each renormalization constant. These RGE's have been derived earlier using a conventional method. ${ }^{8}$ We rederive it because we feel the superfield version is simpler and it illustrates very well our points in the text.

We follow the convention of the textbook by Wess and Bagger. ${ }^{9}$ The only minor change is in the normalization of the vector superfield. Their normalization is more suit- 
able for component reduction. We modified for the convenience of supergraph calculations.

\section{AMBIGUITY IN SUPERFIELD TERMS}

Let us consider the toy Lagrangian

$$
\begin{aligned}
L_{1}= & A^{*} \square A-i \psi \sigma^{m} \partial_{m} \bar{\psi}-g^{2}\left|A A^{*}\right|^{2}+g A \psi^{2} \\
& +g A^{*} \bar{\psi}^{2}+a g \mu\left(A^{3}+A^{* 3}\right)+b \mu^{2} A A^{*} .
\end{aligned}
$$

Here $A$ is a complex scalar field and $\psi$ is the Weyl spinor. The last two terms are SUSY-breaking terms and in their absence the Lagrangian reduces to that of the WessZumino $^{1}(\mathrm{WZ})$ model.

Now we shall express this Lagrangian by superfields. However, before we do that, we shall digress a bit and discuss spurions. Spurions are nondynamical superfields introduced as an artifact for describing supersymmetry breaking in superspace language. It usually is a function of $\theta$ only. Consequently, they break the translation invariance in the superspace. Because of their nondynamical nature they can appear only as the external legs and do not propagate inside the loops in supergraphs. Since the Lagrangian in terms of spurions looks very similar to the SUSY one (SUSY breaking being completely hidden in the explicit value of the spurion field) all supergraph rules can still be applied the same way as in the supersymmetric case. In this way spurions keep the powerful machinery of superfields rolling even when SUSY is explicitly broken. To cast $L_{1}$ in the superfield form we employ a chiral superfield $\Phi(x, \theta, \bar{\theta})=A(x)+\psi(x) \theta+\cdots$ and a

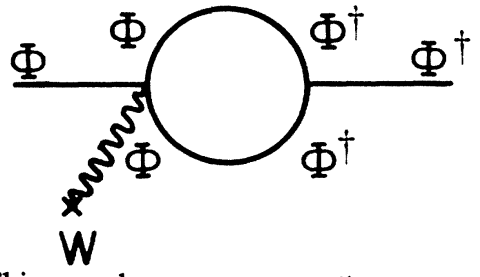

FIG. 1. This graph generates a divergent $\int \Phi^{\dagger} \Phi W d^{4} \theta$ type- $D$ term. The wavy line ending in a cross represents a spurion.

spurion field $W=\mu \theta^{2}$ where $\mu$ characterizes the SUSYbreaking scale and also keeps $W$ dimensionless. The Lagrangian can now be written as

$$
\begin{aligned}
L_{2}= & \int d^{4} \theta \Phi^{\dagger} \Phi+\frac{g}{3}\left(\int d^{2} \theta \Phi^{3}+\text { H.c. }\right) \\
& +a g \mu\left(\int d^{2} \theta W \Phi^{3}+\text { H.c. }\right) \\
& +b \int d^{4} \theta W^{\dagger} W \Phi^{\dagger} \Phi
\end{aligned}
$$

It is obvious that the introduction of an explicit value for $W$ yields the last two SUSY-breaking terms of $L_{1}$. However, this straightforward way of translating the SUSY-breaking terms into superfield form turns out to be too naive. To start with, this Lagrangian is not renormalizable in the sense that it generates a new logarithmically divergent term of the form $\int d^{4} \theta W \Phi \Phi^{\dagger}$ through the graph depicted in Fig. 1. Hence to make it renormalizable we must extend the Lagrangian of Eq. (2) to include this term and arrive at

$L_{3}=\int d^{4} \theta \Phi^{\dagger} \Phi+\left[\frac{g}{3} \int d^{2} \theta \Phi^{3}+\alpha_{1} g \int d^{2} \theta W \Phi^{3}+\right.$ H.c. $)+\alpha_{2} \int d^{4} \theta W^{\dagger} W \Phi^{\dagger} \Phi+\alpha_{3} \int d^{4} \theta\left(W \Phi^{\dagger} \Phi+\right.$ H.c. $)$

A glimpse at this Lagrangian immediately prompts some questions. We started out with only two SUSYbreaking terms in components and ended up with three terms in the superfield form. Apparently all three parameters $\alpha_{1}, \alpha_{2}$, and $\alpha_{3}$ could not be independent physical parameters. There must be some redundancy involved. It is in fact not very hard to get rid of this redundancy. By the nonrenormalization theorem we see that the $F$ term with the coupling constant $\alpha_{1}$ will never be generated in the perturbation theory. We also note that the coefficients $\alpha_{2}$ and $\alpha_{3}$ together are sufficient to generate $a$ and $b$ terms of the component form in Eq. (2). The obvious economical choice is to set $\alpha_{1}=0$. This is the central theme of the paper. In practical calculations, there is a particular choice of terms involving spurions that are more economical

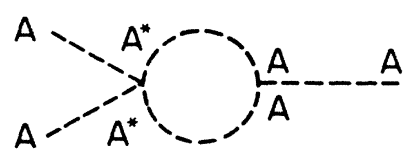

FIG. 2. This graph renormalizes the triscalar coupling $a$. Dashed lines are $\boldsymbol{A}$ field legs. than the others. Also note that, after setting $\alpha_{1}=0$, there are equal numbers of breaking terms in the component and the superfield versions. Even though verified in the last example and expected intuitively the proof of this one-to-one correspondence in general is not that obvious from our discussions so far. In the next section we shall generalize this result to include all soft-breaking terms.

In the remainder of this section we shall simply push this toy example further and compare the RGE's obtained from the component and superfield approaches. Relations

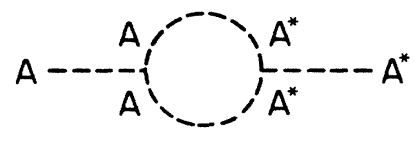

(a)

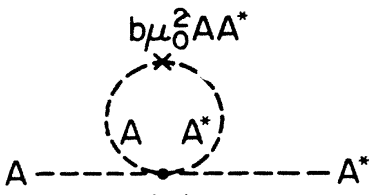

(b)

FIG. 3. These graphs renormalize the coupling $b$. 


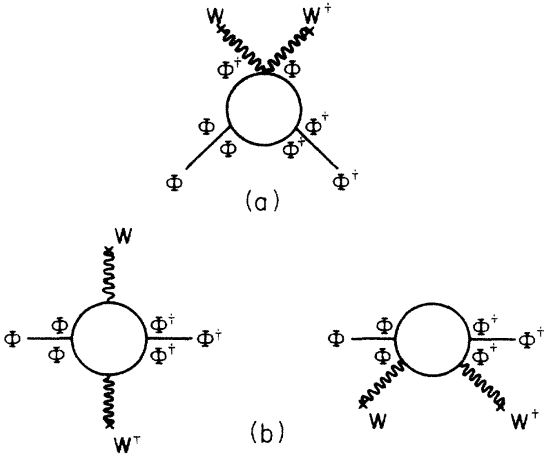

FIG. 4. These graphs renormalize the coupling $\alpha_{2}$. The continuous lines represent chiral superfields.

among $a, b, \alpha_{2}$, and $\alpha_{3}$ can be obtained easily by substituting the value of $W$ and integrating out $\theta$ to arrive at

$$
a=-\alpha_{3}, \quad b=\alpha_{2}-\alpha_{3}{ }^{2} .
$$

Graphs that contribute towards RGE's of $a$ and $b$ are depicted in Figs. 2 and 3, respectively. From them one gets

$$
\begin{aligned}
& \beta_{a}=\mu \frac{\partial a}{\partial \mu}=\frac{12 a}{16 \pi^{2}} g^{2}, \\
& \beta_{b}=\mu \frac{\partial b}{\partial \mu}=\frac{12 a}{16 \pi^{2}} g\left(b-3 a^{2}\right) .
\end{aligned}
$$

Next we shall calculate RGE's for $\alpha_{2}$ and $\alpha_{3}$ using supergraph techniques. Supergraphs that contribute are shown in Figs. 4 and 5. Before we get to their RGE's we would like to share an interesting observation about supergraph calculations. We found that whenever the number of chiral super propagators in a supergraph increases by one unit the sign of the graph contribution changes. For instance, 4(a) has a sign opposite to that of $4(\mathrm{~b})$ and $4(\mathrm{c})$.

Figures 4 and 5 contribute towards the vertex renormalization of constants $\alpha_{2}$ and $\alpha_{3}$, respectively. One finds from them

$$
\begin{aligned}
& \beta_{\alpha_{2}}=\left(\alpha_{2}-2 \alpha_{3}^{2}\right)\left(\frac{12 g^{2}}{16 \pi^{2}}\right), \\
& \beta_{\alpha_{3}}=\left(\frac{12 g^{2}}{16 \pi^{2}}\right) \alpha_{3} .
\end{aligned}
$$

$$
\begin{aligned}
L_{S}= & \int d^{4} \theta \Phi^{\dagger} \Phi+\left[\frac{1}{2} m^{2} \int d^{2} \theta \Phi^{2}+\frac{g}{3} \int d^{2} \theta \Phi^{3}+\text { H.c. }\right)+\left(\int d^{2} \theta\left(\mu \beta_{1} W \Phi^{2}+\beta_{2} W \Phi^{3}\right)+\text { H.c. }\right) \\
& \left.+\iint d^{4} \theta\left(\alpha_{1} W \Phi^{\dagger} \Phi+\frac{1}{2} \alpha_{2} W^{\dagger} W \Phi^{\dagger} \Phi+\mu \alpha_{3} \Phi^{\dagger} W+\mu \alpha_{4} W^{\dagger} W \Phi+\text { H.c. }\right)\right]
\end{aligned}
$$

Here, as before, $\Phi$ are chiral superfields and $W$ is the spurion. $\alpha_{i}$ and $\beta_{i}$ are SSB parameters. Substituting $\mu \theta^{2}$ for $W$ and integrating out $\theta$ and auxiliary fields one obtains the following SUSY-breaking terms:

$$
\begin{aligned}
L_{\mathrm{SSB}}= & \mu^{2} A\left(\alpha_{4} \mu-\alpha_{3} m-\alpha_{1} \alpha_{3} \mu\right) \\
& +\frac{1}{2} \mu^{2}\left(\alpha_{2}-\alpha_{1}{ }^{2}\right) A A^{*} \\
& +\mu A^{2}\left(\beta_{1} \mu-g \mu \alpha_{3}-\alpha_{1} m\right) \\
& +\mu\left(\beta_{2}-\alpha_{1} g\right) A^{3}+\text { H.c. }
\end{aligned}
$$

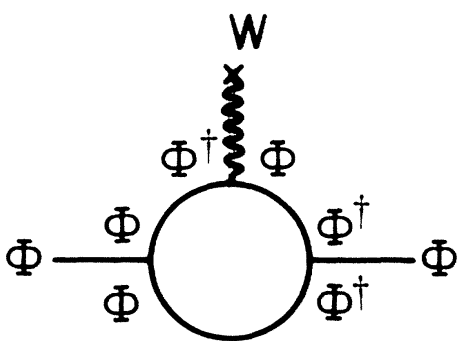

FIG. 5. These graphs renormalize the coupling $\alpha_{3}$.

Using Eqs. (6) and (4) one can calculate $\beta_{a}$ and $\beta_{b}$ and find that they are just the same as those in Eq. (5) derived by the component method. This verifies the equivalence of $L_{1}$ and $L_{3}$ when $\alpha_{1}$ is set equal to zero.

\section{SOFT-SUSY-BREAKING TERMS AS D-TYPE TERMS}

In the previous section we saw that soft-SUSY-breaking (SSB) terms when cast in $D$-type terms greatly simplify the calculations of RGE's by reducing the number of necessary vertices. In this section we shall show that most general SSB's when added to the WZ model can always be written as $D$ terms. For that purpose consider the Lagrangian

$$
\begin{aligned}
L_{C}= & A^{*} \square A-i \bar{\psi} \sigma^{m} \partial_{m} \psi-m^{2}\left(A A^{*}\right)-g^{2}\left|A A^{*}\right|^{2} \\
& +\left(g A \psi^{2}-m g A^{2} A^{*}+\text { H.c. }\right) \\
& +\left(a g \mu A^{3}+c \mu^{2} A^{2}+d \mu^{3} A+\text { H.c. }\right)+\frac{1}{2} b \mu^{2} A A^{*}
\end{aligned}
$$

The above Lagrangian has been obtained by adding all possible SSB terms to the WZ model (we are not considering gauge interactions at this point). The coefficients $a$, $b, c$, and $d$ are dimensionless SUSY-breaking parameters. To write it in superfield form, a priori, we start with the Lagrangian
As is transparent from above, one could set $\beta_{1}=\beta_{2}=0$ and still reproduce all the terms of Lagrangian $L_{C}$. The nonrenormalization theorem then guarantees that these terms will never be generated in perturbation theory. Since all the divergences can be absorbed in $\alpha_{i}$ 's one could derive correct RGE's ignoring all $\beta_{i}$ 's.

However, one point of caution needs to be stated. All $F$-type terms can be dropped only in the presence of SUSY interactions. For instance, one could not discard $\beta_{2}$ in Eq. (8) if $g$ vanished. In this case one could show that 
the $D$ terms $\int d^{4} \theta W \Phi^{\dagger} \Phi$ and $\int W \Phi^{\dagger} d^{4} \theta$ will not be generated with an infinite coefficient and we can take the superfield Lagrangian to have $\beta_{1}, \beta_{2}, \alpha_{2}$, and $\alpha_{4}$ nonvanishing and set $\alpha_{1}=\alpha_{3}=0$. Note that, in this case, when $g=0$ the SUSY-breaking terms $A^{2}$ and $A^{3}$ do not get renormalized. Thus it is possible to break SUSY and still have SUSY-breaking terms nonrenormalized. This observation may have important consequences on model building.

So far we have confined ourselves to non-gauge-type interactions. Gauge theories require much more careful attention. Since explicit breaking of gauge symmetry leads to nonrenormalizability, all SSB must be written in a gauge-invariant form. A realistic model that illustrates this technique beautifully has been worked out in detail in the Appendix.

Thus we see that in the theories with softly broken supersymmetry the RGE's can be derived very easily using supergraph rules if all the breaking terms are written as $D$ terms with spurion. And we also observe that when supersymmetric interactions are present all soft-SUSY breakings can be cast in $D$-type form. When SUSY interactions are absent, as would be the case if $g=0$ in Eq. (9), we find that some $F$-type terms have to be introduced. These mandatory $F$ terms are protected from any renormalization by the nonrenormalization theorem, which implies that, as stated earlier, broken SUSY theories can be constructed such that some of the breaking parameters are not renormalized. Hence the prescription is that one should always write the SUSY breakings as $D$ terms except when they do not produce all the terms of the component description. The latter implies the presence of some coupling constants that do not receive any renormalization even though SUSY is broken.
The theories with gauge interactions require some simple modification which involves writing the breakings as gauge-invariant $D$ terms. To show the applicability of this approach in practical calculations, and also as an example with gauge interactions, we calculate RGE's for a well-known supersymmetric $\mathbf{S U}(3) \times \mathbf{S U}(2) \times \mathbf{U}(1)$ model of electroweak interaction in the Appendix.

\section{CONCLUSION}

Superfields provide a powerful tool for calculations even when supersymmetry is broken. The effect of supersymmetry breaking is represented by spurion superfields. In general there are many different ways a breaking term can be written using spurions. We showed that the labor could be reduced drastically if SUSY breakings are all written as $D$ terms. Also we found that it is possible to construct broken SUSY theories where some of the breaking parameters do not receive any renormalization. We demonstrated the usefulness of the approach by deriving the RGE for a realistic $N=1$ supergravity model in the Appendix.

After this work was done we received a paper by Grisaru and Zanon where superspace methods were employed for a practical calculation. ${ }^{10}$

\section{ACKNOWLEDGMENTS}

We would like to thank Professor R. N. Mohapatra for numerous discussions and encouragement. We would also like to thank Dr. Palash Pal for reading the entire manuscript and suggesting many corrections. One of us (A.G.) thanks the elementary-particle-physics group of University of Maryland for their warm hospitality.

\section{APPENDIX}

As an application of the machinery developed in the text, we shall derive RGE's for a realistic supersymmetric $\mathrm{SU}(3) \times \mathrm{SU}(2) \times \mathrm{U}(1)$ model given by Alvarez-Gaumé, Wise, and Polchinski. ${ }^{11}$ The model is described by the Lagrangian

$$
\begin{aligned}
L= & \sum_{m n}\left(g_{e}^{m n} \hat{E}^{m} \hat{L}^{n} \hat{H}+g_{d}^{m n} \hat{D}^{m} \hat{Q}^{n} \hat{H}+g_{u}^{m n} \hat{U}^{m} \hat{Q}^{n} \hat{H}^{\prime}\right)-m_{3 / 2} \sum_{m n}\left(A_{e}^{m n} g_{e}^{m n} E^{m} L^{n} H+A_{d}^{m n} g_{d}^{m n} D^{m} Q^{n} H+A_{u}^{m n} g_{u}^{m n} U^{m} Q^{n} H^{\prime}\right) \\
& -\sum_{i} m_{i}{ }^{2} \Phi_{i}{ }^{2}+\text { kinetic energy terms }
\end{aligned}
$$

Here a caret over the letter indicates a superfield and those without a caret are the corresponding scalar components. The subscripts $m, n$ are generate indices.

Since this is a gauge theory one must write all SSB's in gauge-invariant form. Using the procedure developed in the text we write the breaking terms in the following form with spurion $W\left(W=\mu \theta^{2}\right)$ :

$$
\begin{aligned}
L_{\mathrm{SSB}}= & \alpha_{1 a} \int d^{4} \theta W^{\dagger} W \Phi^{a+}\left(e^{-2 g \mathrm{~V} \cdot \mathbf{T}}\right) \Phi^{a} \\
& +\alpha_{2 a} \int d^{4} \theta\left(W^{\dagger}+W\right) \Phi^{a+}\left(e^{-2 g \mathrm{~V} \cdot \mathbf{T}}\right) \Phi^{a},
\end{aligned}
$$

where $\Phi^{a}$ stands for different superfields like $\hat{E}, \hat{Q}, \hat{U}, \hat{D}$, $\hat{H}$, and $\hat{H}^{\prime} . T_{i}$ are the gauge generators for the represen- tation of $\Phi^{a}$ and $g$ denotes the gauge coupling constants. Before we proceed further we shall, following Ref. 11, assume that $g_{u}{ }^{33}$ (we shall denote it simply by $g_{u}$ ) is the only Yukawa coupling that should be retained for RGE's as others are much smaller. Integrating out $\theta$ and comparing with Eq. (A1) we get

$$
\begin{aligned}
& m_{H^{\prime}}{ }^{2}=\mu^{2}\left(-\alpha_{1 H^{\prime}}+\alpha_{2 H^{\prime}}{ }^{2}\right), \\
& A_{U}^{m n}=\left(\alpha_{2 H^{\prime}}+\alpha_{2 U}^{m}+\alpha_{2 Q}^{n}\right) .
\end{aligned}
$$

Now we can easily calculate the wave-function renormalization constant (WFC) using supergraph rules. The relevant graphs are shown in Fig. 6. We find $\left(Z_{\Phi^{\circ}}\right.$ 


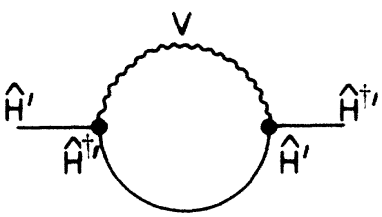

(a)

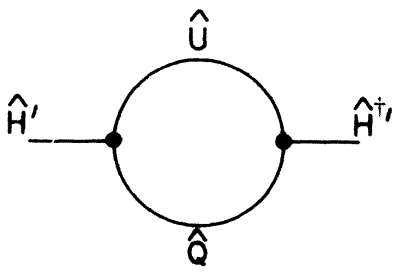

(b)

FIG. 6. These graphs renormalize the superfield $H^{\prime}$.

denotes the WFC for the superfield $\Phi^{a}$ )

$$
\begin{aligned}
& Z_{Q}=1-\left[g_{u}{ }^{2}-\frac{1}{2}\left(\frac{1}{9} g_{1}{ }^{2}+3 g_{2}{ }^{2}+\frac{16}{3} g_{3}{ }^{2}\right)\right] \frac{1}{16 \pi^{2} \epsilon}, \\
& Z_{U}=1-\left[2 g_{u}{ }^{2}-\frac{1}{2}\left(\frac{16}{9} g_{1}{ }^{2}+\frac{16}{3} g_{3}{ }^{2}\right)\right] \frac{1}{16 \pi^{2} \epsilon}, \\
& Z_{H^{\prime}}=1-\left[3 g_{u}{ }^{2}-\frac{1}{2}\left(\frac{1}{9} g_{1}{ }^{2}+3 g_{2}{ }^{2}\right)\right] \frac{1}{16 \pi^{2} \epsilon} .
\end{aligned}
$$

Since $g_{u}$ has no vertex renormalization (coefficient of an $F$-type term) one easily derives the RGE for it by using

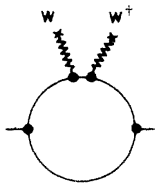

(a)

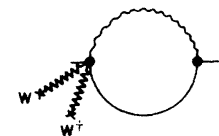

(d)

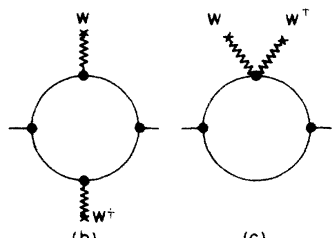

(c)
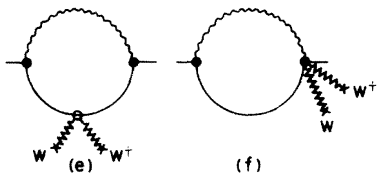

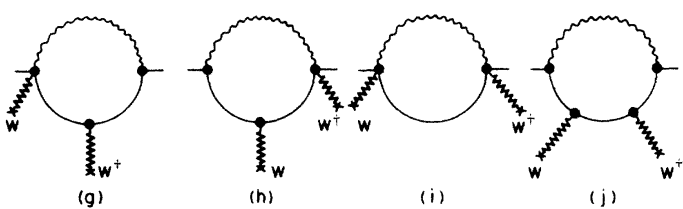

FIG. 7. The wavy line represents a vector superfield. These graphs renormalize the coupling $\alpha_{1 H^{\prime}}$.

$$
\left(g_{u}\right)^{\text {bare }}=\left(g_{u}\right)^{\text {ren }} Z_{H}{ }^{-1 / 2} Z_{U}{ }^{-1 / 2} Z_{Q}{ }^{-1 / 2} .
$$

Differentiating the Eq. (A5) with respect to $\mu$ one gets

$\beta_{g_{u}}=-\epsilon g_{u}+\left(6 g_{u}{ }^{2}-\frac{13}{18} g_{1}{ }^{2}-\frac{3}{2} g_{2}{ }^{2}-\frac{8}{3} g_{3}{ }^{2}\right) \frac{g_{u}}{8 \pi^{2}}$.

Now we shall calculate the vertex renormalization for $\alpha_{1 H^{\prime}}$ and $\alpha_{2 H^{\prime}}$. The graphs that contribute towards $Z_{\alpha_{1 H^{\prime}}}$ and $Z_{\alpha_{2 H^{\prime}}}$ are given in Figs. 7 and 8, respectively. From them we find

$$
\begin{aligned}
Z_{\alpha_{1 H^{\prime}}} & =\text { vertex renormalization for the coefficient } \alpha_{1 H^{\prime}} \\
& =1+\frac{3 g_{u}{ }^{2}}{16 \pi^{2} \epsilon} \frac{\alpha_{1 Q}+\alpha_{1 U}-2\left(\alpha_{2 Q}+\alpha_{2 U}+\alpha_{2 Q} \alpha_{2 U}\right)}{\alpha_{1 H^{\prime}}}+\frac{1}{2} \frac{g_{1}{ }^{2}+3 g_{2}{ }^{2}}{16 \pi^{2} \epsilon} \\
Z_{\alpha_{2 H^{\prime}}} & =1+\frac{3 g_{u}{ }^{2}}{16 \pi^{2} \epsilon} \frac{\alpha_{2 Q}+\alpha_{2 U}}{\alpha_{2 H^{\prime}}}+\frac{1}{2} \frac{g_{1}{ }^{2}+3 g_{2}{ }^{2}}{16 \pi^{2} \epsilon}
\end{aligned}
$$

Using wave-function renormalization constants derived in Eq. (A4), we can easily calculate the following RGE's:

$$
\begin{aligned}
& \mu \frac{\partial \alpha_{1 H^{\prime}}}{\partial \mu}=\frac{3 g^{2}}{8 \pi^{2}}\left[\alpha_{1 Q}+\alpha_{1 U}+\alpha_{1 H^{\prime}}\right. \\
& \left.-2\left(\alpha_{2 Q}{ }^{2}+\alpha_{2 U}{ }^{2}+\alpha_{2 U} \alpha_{2 Q}\right)\right], \\
& \mu \frac{\partial \alpha_{2 H^{\prime}}}{\partial \mu}=\frac{3 g^{2}}{8 \pi^{2}}\left(\alpha_{2 Q}+\alpha_{2 U}+\alpha_{2 H^{\prime}}\right) .
\end{aligned}
$$

Now using the relationship derived in Eq. (A3) one finds the following RGE's for $m_{H^{\prime}}{ }^{2}$ and $A_{U}{ }^{33}$ :

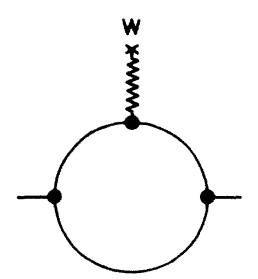

(a)

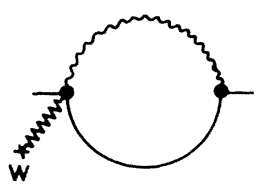

(c)

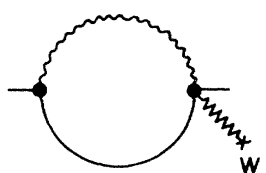

(b)

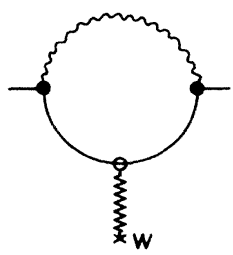

(d)
FIG. 8. These graphs renormalize the coupling $\alpha_{2 H^{\prime}}$. 


$$
\begin{aligned}
& \mu \frac{\partial m^{2}}{\partial \mu}=\frac{3 g^{2}}{8 \pi^{2}}\left(m_{H^{\prime}}{ }^{2}+m_{U^{\prime}}{ }^{2}+m_{Q^{\prime}}{ }^{2}+m_{g}{ }^{2} A_{U}{ }^{33}\right), \\
& \mu \frac{\partial A_{U}{ }^{33}}{\partial \mu}=\frac{6 g^{2}}{8 \pi^{2}} A_{U}{ }^{33} .
\end{aligned}
$$

In a similar way we could derive the RGE's for $m_{Q}{ }^{2}$ and $m_{U}{ }^{2}$. Comparing with Ref. 11 one finds that our approach gives the same answer as derived by the conventional method.
1J. Wess and B. Zumino, Nucl. Phys. B78, 1 (1974); A. Salam and J. Strathdee, Phys. Lett. 51B, 353 (1974); 51B, 475 (1974); Nucl. Phys. B76, 477 (1974); Phys. Rev. D 11, 1521 (1975); S. Ferrara and B. Zumino, Nucl. Phys. B79, 413 (1974); S. Ferrara, J. Iliopoulos, and B. Zumino, Phys. Lett. 51B, 238 (1974); B. A. Ovrut, and J. Wess, Phys. Rev. D 25, 409 (1982). ${ }^{2}$ E. Witten, Nucl. Phys. B185, 153 (1981); R. K. Kaul, Phys. Lett. 109B, 19 (1982).

${ }^{3}$ E. Gildener, Phys. Rev. D 14, 1667 (1976).

${ }^{4}$ A. Salam and J. Strathdee, Phys. Rev. D 11, 1521 (1975).

5S. Ferrara, J. Iliopoulos, and B. Zumino, Phys. Lett. 51B, 239 (1974).
${ }^{6}$ A. Salam and J. Strathdee, Phys. Rev. D 11, 1521 (1975); M. T. Grisaru, M. Rocek, and .W. Siegel, Nucl. Phys. B159, 429 (1979); L. Girardello and M. T. Grisaru, Nucl. Phys. B194, 65 (1982).

${ }^{8}$ K. Inoue, A. Kakuto, H. Komatsu, and S. Takeshita, Prog. Theor. Phys. 68, 927 (1982).

9J. Wess and J. Bagger, Supersymmetry and Supergravity (Princeton University Press, Princeton, 1982).

${ }^{10}$ M. T. Grisaru and D. Zanon, Class. Quant. Gravit. 2, 477 (1985).

${ }^{11}$ L. Alvarez-Gaumé, M. B. Wise, and J. Polchinski, Nucl. Phys. B221, 495 (1983). 